\title{
Disección endoscópica con técnica de bolsillo submucoso. ¿Puede reemplazar a la disección convencional?
}

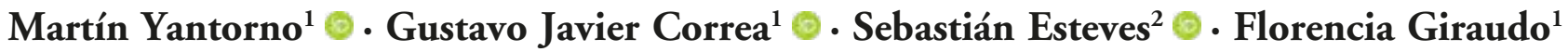

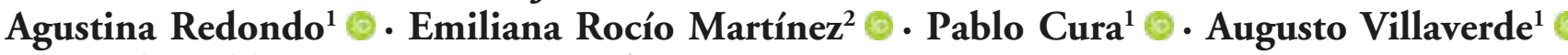 \\ Fernando Baldoni ${ }^{1} \odot$. Francisco Tufare ${ }^{1}$ \\ ${ }^{1}$ Médico especialista en Gastroenterología. Hospital Interzonal General de Agudos "General José de San Martín" de La Plata, Buenos Aires, Argentina. \\ ${ }^{2}$ Médico especialista en Gastroenterología. Clínica CMIC, Neuquén, Argentina.
}

Acta Gastroenterol Latinoam 2021;51(3):327-332

Recibido: 25/06/2021 / Aceptado: 17/08/2021 / Publicado online: 27/09/2021 / https://doi.org/10.52787/ujns2488

\begin{abstract}
Resumen
La disección submucosa endoscópica es una técnica compleja que permite la resección en bloque de lesiones de gran tamaño. Se la asocia a procedimientos técnicamente complejos, de larga duración y alto riesgo de complicaciones. La creación de un bolsillo submucoso es una variante de la técnica convencional que permite reducir estas dificultades, generando una alta tasa de resección completa, con menor tiempo de procedimiento, mayor velocidad de disección y menor tasa de eventos no deseados. Si bien esta variante fue descripta inicialmente en el estómago, su aplicación se ha generalizado a otros sectores del tubo digestivo. Presentamos dos casos donde se utilizó esta variante para el tratamiento de lesiones tempranas de gran tamaño, con éxito técnico.
\end{abstract}

Palabras claves. Neoplasias colorrectales, disección, resección endoscópica de la mucosa, neoplasias gastrointestinales, cirugía.

\section{Endoscopic Dissection with Submu- cosal Pocket Technique. May it place Conventional Dissection?}

\section{Summary}

Endoscopic submucosal dissection is a complex technique that allows en bloc resection of large lesions. It is associated with long-term, technically complex procedures and a high risk of complications. The creation of a submucosal pocket is a variant of the conventional technique that reduces these difficulties, generating a high rate of complete resection with a shorter procedure time, a faster dissection speed and a lower rate of adverse events. Even though this variant was initially described in the stomach, its application has been generalized to other areas of the digestive tract. We present two cases where this variant technique was applied to treat large early lesions, with technical success.

Keywords. Colorectal neoplasms, dissection, endoscopic mucosal resection, gastrointestinal neoplasms, surgery.

\section{Abreviaturas}

DSE: Disección submucosa endoscópica.

RME: Resección mucosa endoscópica.

DSE-TBS: Disección submucosa endoscópica con técnica de creación de bolsillo submucoso. 


\section{Puntos claves}

- DSE convencional asociada a una alta dificultad técnica y complicaciones.

- Variante DSE-TBS como estrategia para mejorar la eficacia y la seguridad.

- En comparación a la DSE convencional demostró menores eventos adversos, mayor velocidad de disección con menor tiempo de procedimiento y mayor tasa de resección en bloque.

\section{Introducción}

La disección submucosa endoscópica (DSE) es una técnica endoscópica avanzada para el tratamiento de lesiones preneoplásicas o neoplásicas tempranas, independientemente de su tamaño y localización. Es un procedimiento complejo, que requiere de la adquisición de habilidades técnicas, con curvas de aprendizajes extensas. ${ }^{1}$

Numerosos estudios señalan que la DSE convencional se asocia a procedimientos de larga duración con un riesgo elevado de complicaciones, como el sangrado y la perforación. ${ }^{1,2}$ La presencia de fibrosis, sumada a la orientación de la lesión al endoscopio y los movimientos respiratorios dificultan las maniobras necesarias para lograr la resección completa, prolongando el tiempo del procedimiento y aumentando el riesgo de eventos no deseados. ${ }^{3}$

Se han reportados distintas alternativas para superar estas dificultades, como los métodos de tracción interna (clips, bandas elásticas) o externa (pinzas, hilo dental), endoscopios con doble canal de trabajo y hasta métodos asistidos por robot. Sin embargo, estas variantes necesitan de dispositivos o equipos adicionales, que pueden resultar en un costo y un inconveniente adicional para el operador. ${ }^{4}$ Con el fin de evitar estas dificultades, se ha descripto una alternativa a la DSE convencional, inicialmente en lesiones gástricas y luego extendida a colon y otros sectores del tracto digestivo: la creación de un bolsillo submucoso (DSE-TBS), que consiste en realizar una incisión mucosa mínima, para entrar en el plano submucoso con el endoscopio, continuando la disección de este plano por debajo de la lesión a modo de bolsillo. Una vez finaliza- do, se amplía en forma lateral y se completa la disección periférica de la circunferencia. Esta alternativa se ha asociado a un menor riesgo de complicaciones y tiempo de procedimiento. ${ }^{3,5,6}$ Además, distintos expertos postulan que requiere curvas de aprendizajes menos dificultosas en comparación con la técnica convencional. Si bien se ha desarrollado en Oriente, aún no cuenta con estudios prospectivos de calidad en Latinoamérica. ${ }^{6}$

Presentamos a continuación dos casos, donde se utiliza esta técnica para la resección de lesiones gastrointestinales.

\section{Caso 1}

Mujer de 58 años, derivada para tratamiento de una lesión plano-elevada con centro deprimido ubicada en la cara posterior del antro gástrico, con biopsias que informaban adenoma con displasia de alto grado. Se evaluó la lesión con gastroscopio (Olympus EVIS EXERA ${ }^{\circledR} 180 \mathrm{H}$ ) utilizando luz blanca y, posteriormente, cromoendoscopía digital con $\mathrm{NBI}^{\circledR}$ (narrow band imaging). Se objetivó una lesión de $30 \mathrm{~mm}$, con cicatriz central asociada a biopsia previa. Por cromoendoscopía digital, se evidenció el límite de demarcación de la lesión con patrón vascular y de superficie irregular, lo cual sugería una alta probabilidad de un cáncer gástrico temprano. Se realizó ultrasonografía endoscópica radial con minisonda de $12 \mathrm{MHz}$ (Olympus $^{\circledR}$ ) descartando invasión de la submucosa. Dadas las características de la lesión y la clasificación de Gotoda, se decidió realizarle una resección en bloque mediante la DSE-TBS. Se le realizó una limpieza gástrica con n-acetilcisteína y simeticona, insuflación con $\mathrm{CO}_{2}$, con administración endovenosa de hioscina para disminuir el peristaltismo gástrico. Se utilizó fuente electroquirúrgica $\mathrm{ERBE}^{\circledR}$ VIO 300 (Tabla 1). Se realizó la marcación de la lesión $3 \mathrm{~mm}$ por fuera, utilizando un knife (Flush knife BTS ${ }^{\circledR}$, FUJI Film ${ }^{\circledR}$ ) y un capuchón punta cónica (ST Hood Fuji Film ${ }^{\circledR}$ ). Se efectuó una incisión en el lado proximal (oral) a modo de ojal, previa elevación con solución coloide $\left(V_{\text {oluven }}{ }^{\circledR}\right.$ ) e índigo carmín. Se accedió al plano submucoso, continuando la disección

Tabla 1. Fuente electroquirúrgica

\begin{tabular}{ll}
\hline Etapa de la DSE & Fuente electroquirúrgica \\
\hline Marcación & Soft coag, E4, 60-100 W \\
Incisión "ojal" & ENDOCUT I, E2-4, D1-3, I1-3 \\
Disección submucosa & Coagulación forzada, Swit coag, Dry Cut, E2-3, 35-100W \\
Hemostasia (pinza hemostática) & Soft coag, E5, 60-100 W \\
\hline
\end{tabular}


por debajo de la lesión a modo de bolsillo (Tabla 1). Se coagularon pequeños vasos con la punta del knife y los de mayor calibre con la pinza hemostática (Coagrasper, Olympus $^{\circledR}$ ) y coagulación suave. Se continuó el bolsillo hasta observar, desde la luz gástrica, la coloración azulada en el lado distal de la lesión. Una vez completado, se disecaron los bordes laterales utilizando un chuchillo de punta aislada (IT-knife Olympys ${ }^{\circledR}$ ) (ver Figura 1, Video 1). La lesión fue recuperada. No se observaron signos de injuria muscular profunda. El tiempo total del procedimiento fue de 47 minutos. La paciente inició la alimentación oral a las $24 \mathrm{~h}$ del procedimiento con buena tolerancia y fue dada de alta a las $48 \mathrm{~h}$, bajo tratamiento con inhibidor de la bomba de protones.

El estudio histopatológico informó una lesión de 30 x 28 x $6 \mathrm{~mm}$ en la que se evidenció un adenoma con displasia epitelial de alto grado y metaplasia intestinal completa focal, con márgenes de resección libres de displasia.

Se realizaron controles endoscópicos a los 3 y 6 meses, evaluando cicatriz con luz blanca y cromoendoscopía digital, sin evidenciar lesión y con biopsias de la cicatriz sin recurrencia microscópica.

Figura 1. Imágenes endoscópicas de la DSE-TBS para lesión gástrica
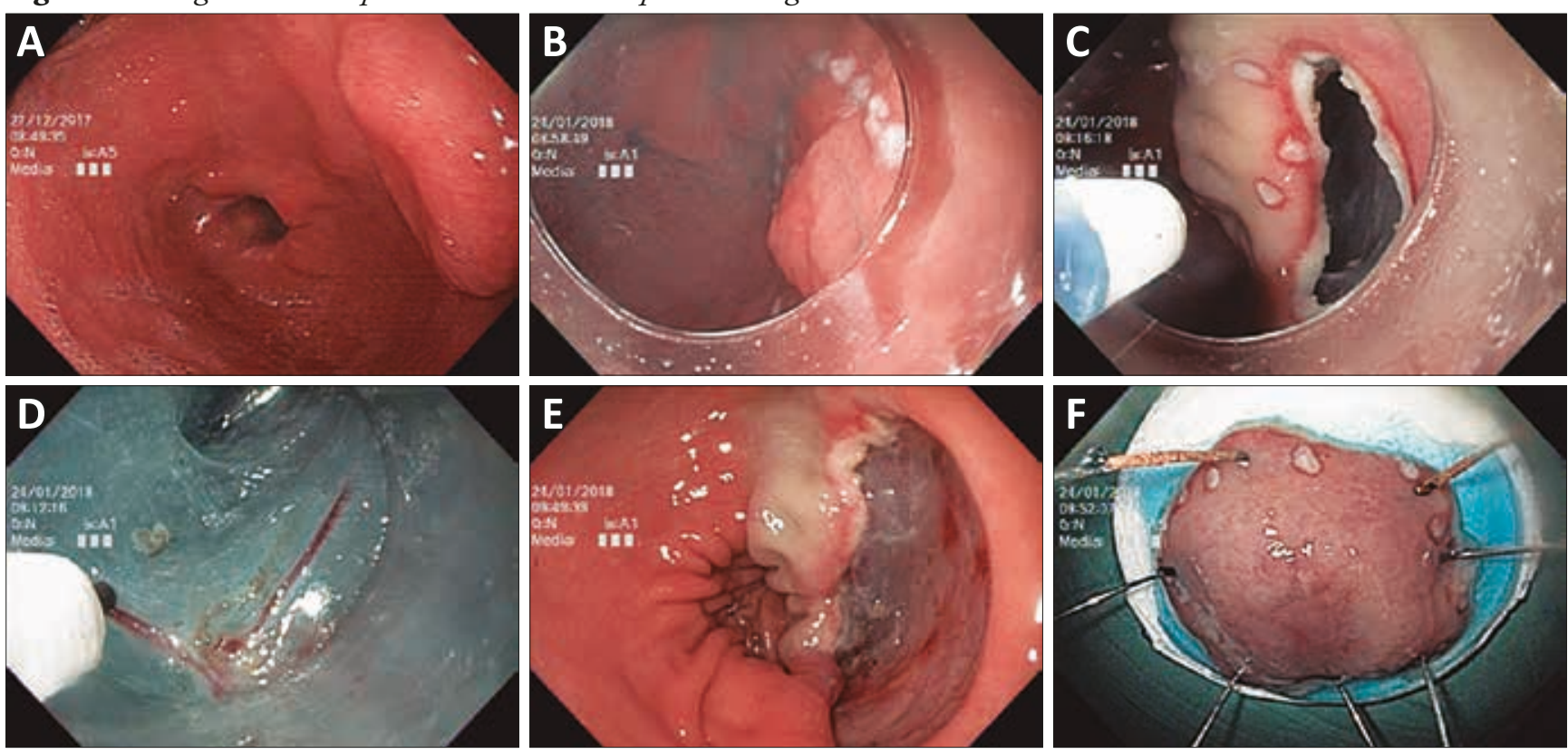

A) Evaluación con luz blanca. B) Marcación de la lesión. C) Incisión proximal, a modo de ojal, para ingresar al bolsillo submucoso. D) Coagulación de vasos de pequeño calibre con punta del knife, dentro del bolsillo submucoso. E) Lecho de resección. F) Extendido de la pieza resecada.

Video 1. Caso de la DSE-TBS gástrica

https://www.youtube.com/watch? $=Y V n 7 A 9 z N 1$ ls\&ab_channel=ActaSage

\section{Caso 2}

Paciente femenina de 62 años. Se le realiza una videocolonoscopía por un dolor abdominal. Se observa, en el recto medio, una lesión de crecimiento lateral no granular, pseudodeprimida, de $30 \mathrm{~mm}$ de diámetro. Fue evaluada bajo visión con luz blanca, cromoendoscopía vital con índigo carmín y digital con i-Scan ${ }^{\circledR}\left(\right.$ PENTAX $\left.^{\circledR}\right)$, clasificándose como Kudo IIIs (a nivel del centro de la lesión) y con patrón vascular y de superficie irregular. Dado que presentaba signos sugestivos de invasión submucosa superficial, se decidió realizarle la resección en bloque, mediante la DSE-TBS. Se utilizó una pre- paración colónica con polietilenglicol, un gastroscopio (PENTAX ${ }^{\circledR}$ Serie EG29-i10) e insuflación con $\mathrm{CO}_{2}$. Se utilizó una fuente electroquirúrgica $\mathrm{ERBE}^{\circledR}$ VIO 300 (Tabla 1). Se realizó la marcación por fuera de la lesión con knife (Flush knife BTS, FUJI Film ${ }^{\circledR}$ ), elevación con solución coloide $\left(\right.$ Voluven $\left.^{\circledR}\right)$ e índigo camín y un ojal en el sector distal (anal). Con la ayuda de un capuchón de punta cónica (ST Hood FUJI Film ${ }^{\circledR}$ ), se ingresó al plano submucoso y se comenzó la disección en forma de bolsillo, hacia el sector proximal (cecal) de la lesión. Se coagularon vasos utilizando la técnica de coagulación suave con la punta del knife en aquellos vasos de peque- 
ño calibre y con pinza hemostática los de mayor tamaño. Una vez completado el bolsillo, al evidenciar el color azulado desde la luz rectal sobre su sector proximal, se realizó la disección lateral, con capuchón blando, completando la resección en bloque de la lesión. El tiempo de disección fue de 104 minutos (ver Figura 2, Video 2). La paciente permaneció en observación y presentó, a las 24 horas del procedimiento, un sangrado tardio que fue controlado exitosamente por via endoscópica con la colocación de un clip.

El análisis histológico informó una lesión serrada sesil ( $42 \mathrm{~mm} \times 28 \mathrm{~mm} \times 4 \mathrm{~mm}$ ) con focos de displasia epitealial de alto grado, con bordes en profundidad y laterales libres de lesión.

Se le realizó un control endoscópico a los 3 meses, sin signos macroscópicos ni microscópicos de recurrencia.

\section{Figura 2. Imágenes endoscópicas de la DSE-TBS del recto}
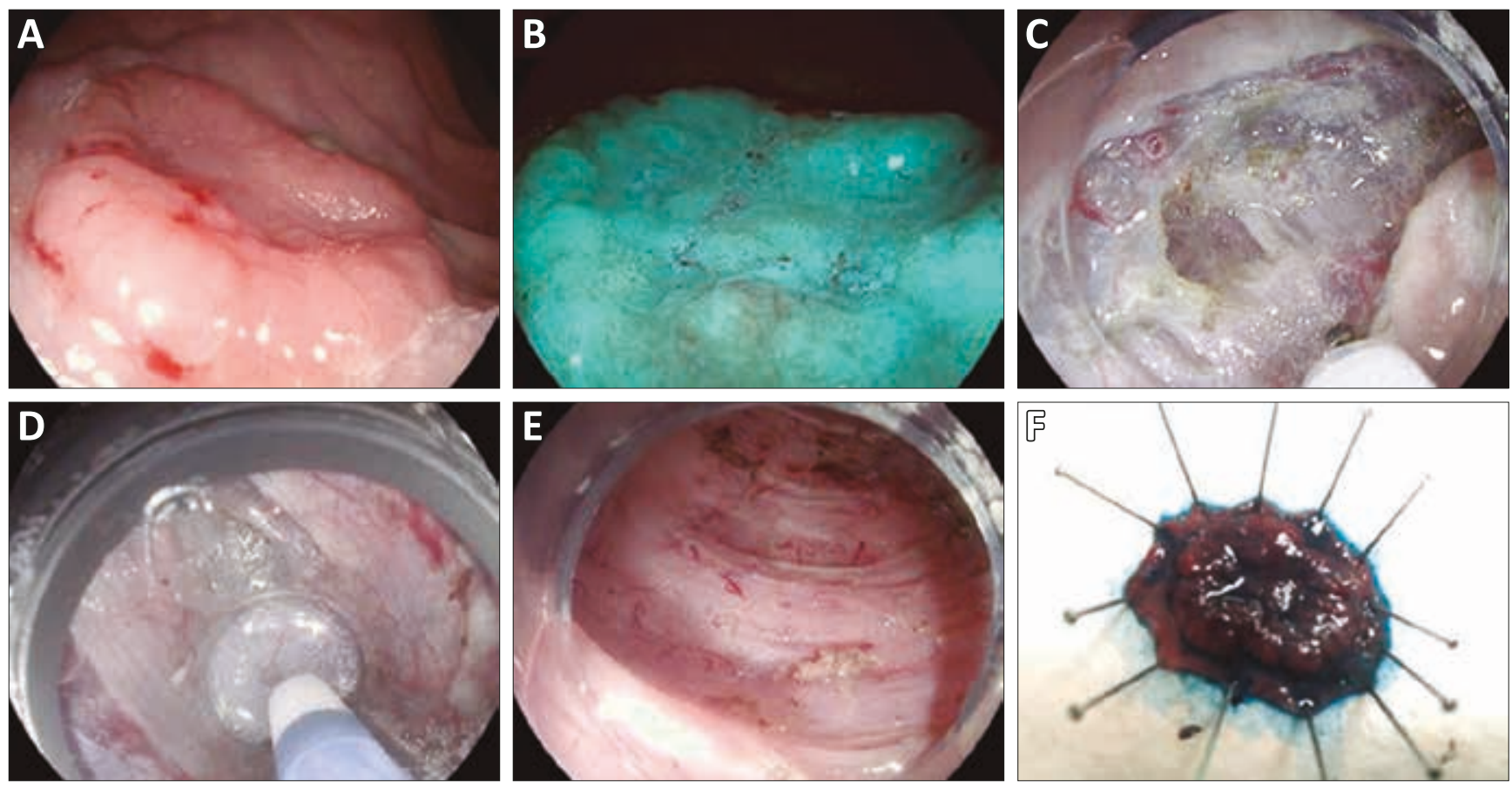

A) Evaluación con luz blanca. B) Evaluación con cromoendoscopía digital. C) Incisión proximal, a modo de ojal, para ingresar al bolsillo submucoso. D) Disección del bolsillo submucoso E) Lecho de resección. F) Extendido de la pieza resecada.

Video 2. Caso de la DSE-TBS en el recto

https://www.youtube.com/watch? $v=5$ Whca_NaXOE\&feature=youtu.be

\section{Discusión}

La técnica de creación del bolsillo submucoso surge como una alternativa a la disección convencional, mostrando ser eficaz y segura para todo el tracto gastrointestinal. Yamamoto y colaboradores describen este procedimiento a partir de la modificación de la técnica del túnel submucoso, la cual priorizaba la disección submucosa sobre la incisión de la mucosa., 8

Esta variante presenta varias ventajas en comparación con la DSE convencional: permite mantener una capa submucosa amplia con una mínima incisión en la mucosa, lo que evita la fuga de la solución inyectada y proporciona un mejor plano de disección. También, facilita la tracción tisular, lo que resulta en una disección submucosa rápida y la visualización estable de la muscular, incluso ante la presencia de fibrosis severa. Cuando el endoscopio se introduce en el bolsillo creado, alivia los problemas asociados con el movimiento respiratorio, de los latidos cardíacos o del peristaltismo al sincronizar el endoscopio en el bolsillo, mientras se mantiene una buena maniobrabilidad. La DSE con la creación del bolsillo submucoso facilita el reconocimiento de la muscular propia ajustando su ángulo de aproximación, lo que resulta en una línea de disección segura y apropiada, justo por encima de la muscular propia. Esto minimiza el daño tisular debido a la termocoagulación en la muestra resecada. Estos facto- 
res facilitan la resección de una capa submucosa gruesa con un menor trauma tisular, asegurando una evaluación patológica precisa. $3,7,9,10$

Los estudios en distintos órganos (esófago, estómago, duodeno y colon) mostraron buenos resultados, con menor tiempo de procedimiento, mayor tasa de resección R0 y ventajas en el tratamiento de la fibrosis.

En el primer caso, se realizó la resección de una lesión plano-elevada con centro deprimido, ubicada en la cara posterior del antro gástrico, con un tamaño de $30 \mathrm{~mm}$. Se logró la resección en bloque en un tiempo estimado de 47 minutos y sin complicaciones. La evaluación histológica demostró márgenes laterales y en profundidad libres de lesión. Se calculó el área de la muestra resecada con la siguiente fórmula: eje mayor $(\mathrm{mm}) / 2 \mathrm{x}$ eje menor $(\mathrm{mm}) / 2 \times 3,14$. El tiempo de disección fue definido con el tiempo entre la incisión inicial de la mucosa y la finalización de la resección. Se calculó la velocidad de disección con la siguiente fórmula: el área de la muestra $\left(\mathrm{mm}^{2}\right)$ / tiempo de disección (minutos). ${ }^{9}$ En este caso, la superficie resecada fue de $659,4 \mathrm{~mm}^{2}$, con una velocidad de disección de 14,02 $\mathrm{mm}^{2} /$ minuto; con controles endoscópicos posteriores libres de lesión.

Zhang y colaboradores compararon la DSE convencional con la DSE-TBS para lesiones grandes de la curvatura menor gástrica, en un total de 87 procedimientos. La DSE-TBS evidenció mayores tasas de velocidad de disección $\left(8,0 \mathrm{~mm}^{2} / \mathrm{min}\right.$ vs. $\left.7,8 \mathrm{~mm}^{2} / \mathrm{min}, p<0,01\right)$, de resección en bloque ( $100 \%$ vs. $87,3 \%, p=0,035)$ y de resección curativa ( $100 \%$ vs. $85,5 \%, p=0,024)$ en comparación con el grupo DSE convencional. No se produjo perforación o lesión muscular en el grupo DSE-TBS y su tasa de hemorragia intraprocedimiento fue menor (59, $4 \%$ vs. $100 \%, p<0,01) .{ }^{11}$

La DSE colorrectal se considera más difícil que la gástrica. Esto se relaciona, por un lado, a la presencia de una pared colorrectal más delgada que el estómago y, por otro lado, a la dificultad de maniobrar el endoscopio debido a la presencia de pliegues, angulaciones fisiológicas, peristaltismo y movimientos respiratorios. Es importante aclarar que el recto es un segmento fijo, sin haustras y angulación, lo que permite mantener más estable el endoscopio.

En el segundo caso que presentamos, se logró realizar una resección R0 sobre una lesión rectal de crecimiento lateral, con un componente deprimido asociado, con éxito técnico. Se presentó un sangrado posterior que fue resuelto satisfactoriamente. El estudio histopatológico evidenció márgenes de resección libres de lesión. El área resecada fue de $913,16 \mathrm{~mm}^{2}$ y se logró realizar el procedimiento en un tiempo de 104 minutos con una velocidad de disección de $8,87 \mathrm{~mm}^{2} /$ minuto.
Diferentes estudios han evaluado los resultados de la variante de DSE-TBS frente a la técnica convencional, con resultados favorables. Yamamoto y colaborados compararon la técnica convencional con el método de creación de bolsillo, en un total de 126 lesiones de crecimiento granular no homogéneos (LST-NG). En 73 realizaron DSE-TBS y en 53 DSE con técnica convencional. Mostraron una mayor tasa de resección en bloque (100\% DSE-TBS vs. 92\% DSE convencional, $p=0,03$ ) y una velocidad de disección más rápida para el grupo de DSE-TBS (mediana de 9 [13-24] vs. 14 [10-22] $\mathrm{mm}^{2} /$ $\min , p=0,03) .{ }^{12}$ Yamashina y colaboradores realizaron una revisión retrospectiva de 90 grandes tumores colorrectales sésiles en 89 pacientes sometidos a la DSE. Las tasas de resección en bloque y resección R0 fueron similares, pero con la DSE-TBS aumentó la tasa de márgenes verticales negativos de forma considerable. El tiempo de disección fue significativamente más corto y la velocidad de disección fue más rápida en el grupo de la DSETBS. No hubo diferencias significativas en la incidencia de eventos adversos (perforación intraprocedimiento y hemorragia tardía). ${ }^{13}$ Un estudio retrospectivo evaluó la seguridad y efectividad de la DSE-TBS en lesiones colorrectales, comparando 47 pacientes que se trataron con la DSE-TBS, con 49 tratados con técnica convencional: se evidenciaron tasas de resección en bloque más altas $(100 \%$ vs. $84 \% p=0,015)$ y resección endoscópica curativa $(100 \%$ vs. $84 \% p=0,0030)$ en el grupo tratado con la DSE-TBS. No hubo diferencias significativas en las tasas de efectos adversos. Sin embargo, el grupo de la DSE-TBS tuvo menor incidencia de perforación. ${ }^{14}$

Recientemente, Pei Qy colaboradores publicaron un metaanálisis con un total de 1481 pacientes, demostrando que la DSE-TBS logra una tasa de resección R0 más alta $(93,5 \%$ vs. $78,1 \%$; odds ratio [OR] 3,4; intervalo de confianza [IC] 95\% [1,3-8,9]; I2 = 58\%), una mayor tasa de resección en bloque (99,8\% vs. 92,8\%; OR 9,9; IC 95\% [2,7-36,2]; $\left.\mathrm{I}^{2}=0\right)$, con un menor tiempo de procedimiento, una velocidad de disección más rápida y una tasa global de eventos adversos más baja ( $4,4 \%$ vs $6,6 \%$; OR 0,6; IC 95\% [0,3-1,0]; $\left.\mathrm{I}^{2}=0\right)$, en comparación con la DSE convencional. ${ }^{15}$

Por lo tanto, la evidencia disponible señala que la DSE-TBS permite mejorar la calidad de la DSE al aumentar la tasa de resección en bloque, mejorar la velocidad de disección (sin aumentar la incidencia de los efectos adversos) y facilitar la superación de las dificultades asociadas.

En ambos casos, se logró una resección completa de las lesiones, con tiempos cortos de procedimiento y $\sin$ complicaciones mayores. 


\section{Conclusión}

La DSE-TBS es una alternativa eficaz y segura, que disminuye el riesgo de complicaciones y de complejidad técnica para lesiones gastrointestinales. Existen pocas publicaciones al respecto en centros de Latinoamérica, en general, y en Argentina, en particular. La correcta selección de las lesiones, basada en una semiología endoscópica correcta, y el entrenamiento continuo en estas técnicas avanzadas son de gran importancia para poder brindar tratamiento endoscópico curativo en este tipo de lesiones. Futuros estudios serán necesarios para determinar si esta estrategia será considerada como la variante de primera línea para el abordaje endoscópico de lesiones de gran tamaño.

Conflicto de intereses. Los autores declaran que en el presente trabajo de investigación no se presentaron conflictos de intereses.

Sostén financiero. El presente estudio no contó con ningún aporte financiero por parte de ninguna institución en particular.

Consentimiento para la publicación. Para la confección de este manuscrito, se utilizaron datos anonimizados que no han distorsionado su significado científico.

Propiedad intelectual. Los autores declaran que los datos, las figuras y las tablas presentes en el manuscrito son originales y fueron realizados en sus instituciones pertenecientes.

\section{Aviso de derechos de autor}

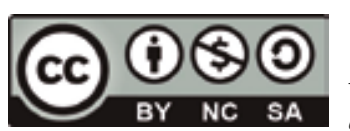

(C) 2021 Acta Gastroenterológica Latinoamericana. Este es un artículo de acceso abierto publicado bajo los términos de la Licencia Creative Commons Attribution (CC BY-NC-SA 4.0), la cual permite el uso, la distribución $y$ la reproducción de forma no comercial, siempre que se cite al autor y la fuente original.

Cite este artículo como: Yantorno M, Correa GJ, Esteves $S$ y col. Disección endoscópica con técnica de bolsillo submucoso. ¿Puede reemplazar a la disección convencional? Acta Gastroenterol Latinoam. 2021;51(3):327-32. https:/ldoi. org/10.52787/ujns 2488

\section{Referencias}

1. Tanaka S, Kashida H, Saito Y, Yahagi N, Yamano H, Saito S, et al. Japan Gastroenterological Endoscopy Society guidelines for colorectal endoscopic submucosal dissection/endoscopic mucosal resection. Dig Endosc. 2020;32(2):219-39.

2. Bourke MJ, Neuhaus H, Bergman JJ. Endoscopic Submucosal Dissection: Indications and Application in Western Endoscopy Practice. Gastroenterology. 2018;154(7):1887-900.e5.

3. Miura Y, Hayashi Y, Lefor AK, Osawa H, Yamamoto H. The pocket-creation method of ESD for gastric neoplasms. Gastrointest Endosc. 2016;83(2):457-8.

4. Kume K. Endoscopic therapy for early gastric cancer: standard techniques and recent advances in ESD. World J Gastroenterol. 2014;20(21):6425-32.

5. Takezawa T, Hayashi Y, Shinozaki S, Sagara Y, Okada M, Kobayashi $Y$, et al. The pocket-creation method facilitates colonic endoscopic submucosal dissection (with video). Gastrointest Endosc. 2019;89(5):1045-53.

6. Yamamoto H. Endoscopic Submucosal Dissection of Early Cancers and Large Flat Adenomas. Clin Gastroenterol Hepatol. 2005;3(7):S74-6.

7. Yamamoto H, Kawata H, Sunada K, Sasaki A, Nakazawa K, Miyata T, et al. Successful en-bloc resection of large superficial tumors in the stomach and colon using sodium hyaluronate and small-caliber-tip transparent hood. Endoscopy. 2003;35(8):690-4.

8. Yamamoto H, Miura Y, Shinozaki S, Hayashi Y, Sakamoto H, Lefor AK. Reply to Tan et al. Endoscopy. 2017;49(4):401.

9. Takezawa T, Hayashi Y, Shinozaki S, Sagara Y, Okada M, Kobayashi $\mathrm{Y}$, et al. The pocket-creation method facilitates colonic endoscopic submucosal dissection (with video). Gastrointest Endosc. 2019;89(5):1045-53.

10. Castillo Bustamante FA, Makoto Sakai C, Horneaux de Moura EG, Sakai P. Técnica de disección endoscópica de "bolsillo submucoso" modificada. Experiencia en porcinos. Acta Gastroenterol Latinoam 2017;47(2):106-9.

11. Zhang X, Shi D, Yu Z, Li R, Chen W, Bai F, et al. A multicenter retrospective study of endoscopic submucosal tunnel dissection for large lesser gastric curvature superficial neoplasms. Surg Endosc. 2019;33(6):1910-9.

12. Sakamoto H, Hayashi Y, Miura Y, Shinozaki S, Takahashi H, Fukuda H, et al. Pocket-creation method facilitates endoscopic submucosal dissection of colorectal laterally spreading tumors, non-granular type. Endosc Int Open. 2017;5(2):E123-9.

13. Yamashina T, Nemoto D, Hayashi Y, Fukuda H, Okada M, Takezawa $\mathrm{T}$, et al. Prospective randomized trial comparing the pocket-creation method and conventional method of colorectal endoscopic submucosal dissection. Gastrointest Endosc. 2020;92(2):368-79.

14. Kanamori A, Nakano M, Kondo M, Tanaka T, Abe K, Suzuki $\mathrm{T}$, et al. Clinical effectiveness of the pocket-creation method for colorectal endoscopic submucosal dissection. Endosc Int Open. 2017;5(12):E1299-305.

15. Pei Q, Qiao H, Zhang M, Wang G, Feng H, Pan J, et al. Pocket-creation method versus conventional method of endoscopic submucosal dissection for superficial colorectal neoplasms: a meta-analysis. Gastrointest Endosc. 2021;93(5):1038-46.e4. 